\title{
NUCLEAR FRÉCHET LATTICES
}

\section{ANTONIO FERNÁNDEZ and FRANCISCO NARANJO}

\author{
(Received 8 November 2000; revised 2 May 2001)
}

Communicated by A. Pryde

\begin{abstract}
We give a characterization of nuclear Fréchet lattices in terms of lattice properties of the seminorms. Indeed, we prove that a Fréchet lattice is nuclear if and only if it is both an AL- and an AM-space.

2000 Mathematics subject classification: primary 46A04, 46A11, 46A40, $06 \mathrm{~F} 30$.

Keywords and phrases: Fréchet lattice, generalized AL-spaces, generalized AM-spaces, nuclear spaces, Köthe generalized sequences spaces.
\end{abstract}

\section{Introduction and preliminaires}

Since its introduction by Grothendieck [5], nuclear spaces have been intensively studied, and nowadays their particular structure is very well understood. Main contributions to these facts were made by Pietsch [11] in the sixties, characterizing nuclear spaces in terms of an intrinsic condition involving a fundamental system of seminorms. Namely, a locally convex space $E$ is nuclear, if and only if for each absolutely convex zero neighbourhood $U$ in $E$ there exist an absolutely convex zero neighbourhood $V$ and a measure $\mu$ on the $\sigma^{*}$-compact set $V^{\circ}$, so that

$$
\|x\|_{U} \leq \int_{v^{0}}\left|\left\langle x, y^{\prime}\right\rangle\right| d \mu\left(y^{\prime}\right)
$$

for all $x \in E$.

At the same time the structure of nuclear Fréchet lattices was studied, amongst others, by Kömura and Koshi [8], Popa [12], Wong [13, 14] and Wong and Ng [15].

This research has been partially supported by La Consejería de Educación y Ciencia de la Junta de Andalucía and the DGICYT project no. PB97-0706.

(C) 2002 Australian Mathematical Society $1446-7887 / 2000 \$ A 2.00+0.00$ 
Especially, Popa showed the following: Let $E$ be a locally convex vector lattice that is topologically complete; then $E$ is nuclear if and only if it has a fundamental system of neighbourhoods $\mathscr{U}$ such that $\widehat{E}_{U}$ is an (AL)-space with a weak order unit for each $U \in \mathscr{U}$, and a fundamental system of neighbourhoods $\mathscr{V}$ such that $\widehat{E}_{V}$ is an (AM)-space for each $V \in \mathscr{V}$. The proof of Popa's result uses several deep results from the literature. As a conclusion, he obtains for topologically complete, separable locally convex lattices a characterization of nuclearity involving lattice properties of the seminorms.

In the present note we present a similar characterization in the general context of Fréchet lattices, removing the additional requirement of the existence of a weak order unit and changing the local Banach lattices by lattice conditions on the seminorms. Our proof is more elementary and more direct. Moreover, from our results we make more transparent the structure of nuclear Fréchet lattices. Compare our Lemma 2.4 and Lemma 2.5 with [8, Theorem 4]. In particular, we obtain that every nuclear Fréchet lattice must be separable (or equivalently, there can be no nuclear Fréchet lattices with an uncountable disjoint system of discrete elements), so that, in a certain sense Popa's result characterizing nuclearity in the setting of separable lattices is the best possible.

Let us recall briefly some definitions and preliminaires. A Fréchet lattice $E$ is called an AL-space if its topology can be defined by a family of lattice seminorms $\|\cdot\|$ that are additive in the positive cone, that is, such that

$$
\|x+y\|=\|x\|+\|y\|, \quad \text { for all } x, y \in E^{+} .
$$

A remarkable property of AL-spaces is that their topology is the so-called Dieudonné topology, that is, the topology of uniform convergence on order-intervals of its dual space (see [13, Corollary 4.2]). In fact, by using the Kantorovic theorem [2, Theorem 1.7] it is not difficult to check that a Fréchet lattice $E$ is an AL-space if and only if for every continuous lattice seminorm $\|\cdot\|$ on $E$ there exists a positive functional $f \in E^{\prime}$ such that $\|x\| \leq\langle f,|x|\rangle$ for all $x \in E$. Another fact that we wish to point out is that every Fréchet AL-space has the Lebesgue property. To check this proceed as in the Banach case considering [4, Theorem 2]. Recall that a Fréchet lattice is said to have the Lebesgue property if whenever a net $x_{\alpha} \downarrow 0$ in the order, then $x_{\alpha} \rightarrow 0$ in the topology. In particular, AL-spaces are order complete (see [1, Theorem 10.3]).

$A$ Fréchet lattice $E$ is called an AM-space if its topology can be defined by a family of lattice seminorms $\|\cdot\|$ such that

$$
\|\sup \{x, y\}\|=\sup \{\|x\|,\|y\|\}, \quad \text { for all } x, y \in E^{+} .
$$

It is not difficult to check that the space $s$ of rapidly decreasing sequences is both an $\mathrm{AL}$ - and an AM-space. On the other hand, it is a very well-known fact, as Kömura and 
Kōmura pointed out, that the space $s$ is a universal generator for all nuclear spaces. Our characterization of nuclear Fréchet lattices says that this is the general situation, that is, we are going to prove that a Fréchet lattice is nuclear if and only if it is both an AL- and an AM-space.

We refer the reader to the excellent books [2] and [10] for the terminology and the unexplained terms used in this paper.

\section{Main result}

Our approach to the main result relies on the setting of Köthe generalized sequences spaces and, in particular, in the use of the Grothendieck-Pietsch condition that characterizes nuclearity of the space $\lambda_{1}(I, A)$ of absolutely summable generalized sequences with respect to a Köthe matrix $A$.

Consider an index set $I$, a priori not countable. An increasing sequence $A=\left(a_{k}\right)_{k}$ of positive families $a_{k}=\left(a_{i, k}\right)_{i \in I}$ is called a Köthe matrix if for each $i \in I$ there exists $k \geq 1$ such that $a_{i, k}>0$. We define

$$
c_{0}(I, A):=\left\{\alpha=\left(\alpha_{i}\right)_{i \in I}: \lim _{i} \alpha_{i} a_{i, k}=0, k=1,2, \ldots\right\}
$$

equipped with the topology generated by the seminorms

$$
\|\alpha\|_{k, \infty}:=\sup \left\{\left|\alpha_{i}\right| a_{i, k}, i \in I\right\} .
$$

Then $c_{0}(I, A)$ is a Fréchet AM-space with the Lebesgue property. To check that $c_{0}(I, A)$ has the Lebesgue property proceed directly from the definition of this property. If the matrix $A$ has all its elements equal to one we simply write $c_{0}(I)$ instead of $c_{0}(I, A)$.

Analogously, we consider

$$
\lambda_{1}(I, A):=\left\{\alpha=\left(\alpha_{i}\right)_{i \in I}: \sum_{i \in I}\left|\alpha_{i}\right| a_{i, k}<\infty, k=1,2, \ldots\right\}
$$

equipped with the topology generated by the seminorms $\|\alpha\|_{k, 1}:=\sum_{i \in I}\left|\alpha_{i}\right| a_{i, k}$. Then $\lambda_{1}(I, A)$ is a Fréchet AL-space. If the matrix $A$ has all its elements equal to one we simply write $\ell_{1}(I)$ instead of $\lambda_{1}(I, A)$.

Observe also that the inclusion $\lambda_{1}(I, A) \subset c_{0}(I, A)$ is a continuous lattice homomorphism.

On the other hand, as indicated by the work of Kōmura and Koshi, our setting must be in the context of a discrete Fréchet lattice.

LEMMA 2.1 ([8, Theorem 3]). Every nuclear Fréchet lattice is discrete. 
Recall that an element $e \neq 0$ of a Fréchet lattice is called discrete (an atomic element in the terminology used by Kōmura and Koshi in [8]) if it follows from $e=u+v$ with $\inf \{|u|,|v|\}=0$ that $u=0$ or $v=0$. A Fréchet lattice is said to be discrete if it coincides with the band generated by all its discrete elements.

REMARK 2.1. (1) According to [9, Theorems 26.4 and 26.8] a discrete element $e$ of a Fréchet lattice is an element whose generating ideal (band) equals the vector subspace generated by $e$.

(2) By using Zorn's lemma we can choose in every discrete Fréchet lattice a maximal disjoint system $\left\{e_{i}\right\}_{i \in I}$ consisting of positive discrete elements, that is, $\inf \left\{e_{i}, e_{j}\right\}=0$, if $i \neq j$ and if $\inf \left\{x, e_{i}\right\}=0$ for all $i \in I$, then $x=0$. Then, following [1, page 17], we see that a Fréchet lattice $E$ is discrete if and only if for every $x \in E$, with $x>0$, there exists a discrete element $e$ of $E$ satisfying $0<e \leq x$.

(3) The spaces $\lambda_{1}(I, A)$ and $c_{0}(I, A)$ are both discrete.

The proof of our main result is based on several lemmas that we now formulate.

LEMMA 2.2. Let E be a Fréchet AM-space with the Lebesgue property. Then $E$ is discrete.

Proof. Assume by contradiction that $E$ is non-discrete. According to Remark 2.1 there exists $x \in E$ such that $x>0$ and there is no discrete element in the order interval $[0, x]$. Take a continuous lattice seminorm $\|\cdot\|$ on $E$ verifying (2) and such that $\|x\|>0$. Since $x$ is a non-discrete element there must exist two disjoint elements $0<y, z<x$ such that $x=y+z$, in which case $x=\sup \{y, z\}$. Thus, we have $\|x\|=\sup \{\|y\|,\|z\|\}$. Accordingly, the set $A:=\{y \in E: 0<y<x,\|y\|=\|x\|\}$ is non-empty. Clearly $A$ is bounded from below. Since $E$ has the Lebesgue property it is order complete and so $v:=\inf A \geq 0$ exists. The set $\{y\}_{y \in A}$, endowed with the order inherited from $E$ is a net for which $\{y\}_{y \in A} \downarrow v$. Since $E$ has the Lebesgue property this convergence is topologically, and so $\|v\|=\lim _{y \in A}\|y\|=\|x\|>0$. This shows that $v \in A$. Now observe that $v$ is a non-discrete element. Repeating the previous argument there will exist an element $z \in E, 0<z<v$ such that $\|z\|=\|v\|=\|x\|$, so that $z \in A$ contradicting that $v$ is the infimum of $A$.

LEMMA 2.3. Let $E$ be a discrete Fréchet lattice with the Lebesgue property and let $\left\{e_{i}\right\}_{i \in I}$ be a maximal disjoint system of discrete elements in $E$. Then every element $x \in E$ can be written as $x=\sum_{i \in I} \alpha_{i}(x) e_{i}$, where the family $\left(\alpha_{i}(x)\right)_{i \in I}$ is uniquely determined by the element $x$.

PROOF. It is enough to prove the decomposition as stated in the lemma for positive elements only. Then, fix $x \geq 0$ in $E$ and consider the family $\mathscr{P}_{f}(I)$ of all finite 
subsets of $I$ ordered by inclusion. Denote by $P_{i}$ the order projection onto the band $E_{i}$ generated by the element $e_{i}$. Since $E_{i}$ coincides with the subspace generated by $e_{i}$, the projection $P_{i}$ is given by $P_{i}(y)=\alpha_{i}(y) e_{i}$ for a certain scalar $\alpha_{i}(y)$ which is uniquely determined by the element $y \in E$. From the positivity of the projections and the disjointness of the bands, we conclude that $\left(\sum_{i \in J} \alpha_{i}(x) e_{i}\right)_{J \in \mathscr{P}_{f}(l)}$ is a positive increasing net order-bounded by $x$. By the Lebesgue property, it follows that the limit

$$
\sum_{i \in I} \alpha_{i}(x) e_{i}:=\lim _{J} \sum_{i \in J} \alpha_{i}(x) e_{i}=\sup \left\{\alpha_{i}(x) e_{i}: i \in I\right\} \leq x
$$

exists.

Put $x_{0}=\sum_{i \in l} \alpha_{i}(x) e_{i}$ and suppose that $x>x_{0}$. By the maximality of $\left\{e_{i}\right\}_{i \in I}$, there exists $i_{0} \in I$ such that

$$
0<\inf \left\{x-x_{0}, e_{i_{0}}\right\}=\inf \left\{x-\sum_{i \in I} \alpha_{i}(x) e_{i}, e_{i_{0}}\right\} \leq \inf \left\{x-\alpha_{i_{0}}(x) e_{i_{0}}, e_{i_{0}}\right\}=0 .
$$

This contradiction establishes the equality $x=x_{0}$. Thus, we have proved that the series $\sum_{i \in I} \alpha_{i}(x) e_{i}$ converges unconditionally to $x$. We also need to show that this decomposition is unique. In fact, if $\sum_{i \in I} x_{i}=0$ with $x_{i} \in E_{i}$ for all $i$, then the continuity of each projection $P_{j}$ and the disjointness of the bands $E_{i}$ 's, imply that $0=\sum_{i \in I} P_{j}\left(x_{i}\right)=P_{j}\left(x_{j}\right)=x_{j}$ for all $j \in I$. Observe also that

$$
|x|=\sum_{i \in l}\left|\alpha_{i}(x)\right| e_{i},
$$

because $\left|\alpha_{i}(x)\right|=\alpha_{i}(|x|)$ for all $i \in I$ (as $P_{i}$ is a lattice homomorphism).

REMARK 2.2. The preceding lemma is a particular case of a more general result which assures that every Fréchet lattice with the Lebesgue property can be decomposed into an unconditional direct sum of a (generally uncountable) family of mutually disjoint ideals, each one having a weak order unit. See [6] for a proof of this statement in the Banach lattice case.

Ando proved in [3] that a Banach AM-space has order continuous norm (the Lebesgue property in our terminology) if and only if it is lattice isometric to $c_{0}(I)$, for some index set $I$. The next result is the Fréchet version of Ando's result.

LEMMA 2.4. Let $E$ be a Fréchet lattice. Then $E$ is an AM-space with the Lebesgue property if and only if it is lattice isomorphic to a Köthe sequence space $c_{0}(I, A)$.

ProOF. Only the if part needs a proof. Consider an increasing sequence $\left(\|\cdot\|_{k}\right)_{k}$ of lattice seminorms defining the topology of $E$ and satisfying condition (2). Since 
$E$ is an AM-space having the Lebesgue property, according to Lemma $2.2 E$ must be discrete. Let $\left\{e_{i}\right\}_{i \in I}$ be a maximal disjoint system of discrete elements. By Lemma 2.3 we know that every element $x \in E$ can be uniquely written as

$$
x=\sum_{i \in I} \alpha_{i}(x) e_{i}
$$

Define the families $a_{k}:=\left(\left\|e_{i}\right\|_{k}\right)_{i \in l}$, for every $k=1,2, \ldots$ Then $A=\left(a_{k}\right)_{k}$ is a Köthe matrix and we may consider the space $c_{0}(I, A)$. Consider also the map $T: E \rightarrow c_{0}(I, A)$ defined by $T(x):=\left(\alpha_{i}(x)\right)_{i \in I}$. From (4) we have that $T$ is well defined and it is clearly linear. Indeed, we have to prove that $\lim _{i} \alpha_{i}(x) a_{i, k}=0$ for all $k=1,2, \ldots$ but this is clear by the convergence of the series. The injectivity of $T$ follows from the uniqueness of the representation (4). We show that $T$ is onto by proving that for every $\left(\alpha_{i}\right)_{i \in I}$ in $c_{0}(I, A)$ the series $\sum_{i \in I} \alpha_{i} e_{i}$ converges in $E$. Fix $k \geq 1$ and take $\varepsilon>0$. Then there exists a finite subset $J_{0} \subset I$ such that $\left|\alpha_{i}\right| a_{i, k}<\varepsilon$ for all $i \notin J_{0}$. For every finite subset $J \subset I$ such that $J \cap J_{0}=\emptyset$ we have

$$
\begin{aligned}
\left\|\sum_{i \in J} \alpha_{i} e_{i}\right\|_{k} & =\left\|\sum_{i \in J} \alpha_{i} e_{i}\left|\left\|_{k}=\right\| \sum_{i \in J}\right| \alpha_{i} \mid e_{i}\right\|_{k}=\left\|\sup \left\{\left|\alpha_{i}\right| e_{i}: i \in J\right\}\right\|_{k} \\
& =\sup \left\{\left|\alpha_{i}\right|\left\|e_{i}\right\|_{k}: i \in J\right\}=\sup \left\{\left|\alpha_{i}\right| a_{i, k}: i \in J\right\}<\varepsilon,
\end{aligned}
$$

so $x:=\sum_{i \in I} \alpha_{i} e_{i}$ defines an element of $E$. Obviously $T(x)=\left(\alpha_{i}\right)_{i \in I}$. Finally, it is easy to prove that $T$ is a lattice homomorphism by using (3).

LEMMA 2.5. Let $E$ be a Fréchet lattice. Then $E$ is a discrete AL-space if and only if it is lattice isomorphic to a Köthe sequence space $\lambda_{1}(I, A)$.

PROOF. Only the if part needs a proof. Consider an increasing sequence $\left(\|\cdot\|_{k}\right)_{k}$ of lattice seminorms defining the topology of $E$ and satisfying condition (1). Let $\left\{e_{i}\right\}_{i \in I}$ be a maximal disjoint system of discrete elements. By Lemma 2.3 we know that every element $x \in E$ can be written uniquely as $x=\sum_{i \in I} \alpha_{i}(x) e_{i}$. Define the families $a_{k}:=\left(\left\|e_{i}\right\|_{k}\right)_{i \in I}$, for every $k=1,2, \ldots$ Then $A=\left(a_{k}\right)_{k}$ is a Köthe matrix and we may consider the space $\lambda_{1}(I, A)$. Consider also the map $T: E \rightarrow \lambda_{1}(I, A)$ defined by $T(x):=\left(\alpha_{i}(x)\right)_{i \in I}$. From (4) we have that $T$ is well-defined and it is clearly linear. Indeed, if $k \geq 1$ and a finite subset $J \subset I$ are given then

$$
\begin{aligned}
\sum_{i \in J}\left|\alpha_{i}(x)\right| a_{i, k} & =\sum_{i \in J}\left|\alpha_{i}(x)\right|\left\|e_{i}\right\|_{k}=\sum_{i \in J}\left\|\alpha_{i}(x) e_{i}\right\|_{k}=\sum_{i \in J}\left\|\left|\alpha_{i}(x)\right| e_{i}\right\|_{k} \\
& =\left\|\sum_{i \in J}\left|\alpha_{i}(x)\right| e_{i}\right\|_{k}=\left\|\sum_{i \in J} \alpha_{i}(x) e_{i} \mid\right\|_{k}=\left\|\sum_{i \in J} \alpha_{i}(x) e_{i}\right\|_{k},
\end{aligned}
$$

and hence $\|T(x)\|_{k, 1}=\|x\|_{k}$ for all $x \in E$. Moreover, it is clear that $T$ is onto. 
The following lemma was proved by Kömura and Koshi. We present here a sketch of its proof for the sake of completeness.

LEMMA 2.6 ([8, Theorem 1]). Every nuclear Fréchet lattice is an AL-space.

PROOF. If $\|\cdot\|$ is a continuous lattice seminorm on $E$ and we denote by $U$ its unit ball, then there exist an absolutely convex zero neighborhood $V$ and a measure $\mu$ on the $\sigma^{*}$-compact set $V^{\circ}$, so that $\|x\| \leq \int_{v^{\circ}}\left|\left\langle x, y^{\prime}\right\rangle\right| d \mu\left(y^{\prime}\right)$, for all $x \in E$. It is then easy to see that the formula

$$
\langle x, g\rangle:=\int_{V^{\circ}}\left\langle x,\left|y^{\prime}\right|\right\rangle d \mu\left(y^{\prime}\right), \quad x \in E,
$$

defines a positive linear functional $g$ on $E$ such that $\|x\| \leq\langle|x|, g\rangle$, for all $x \in E$. Accordingly, $E$ is an AL-space.

LEMMA 2.7 (Grothendieck-Pietsch condition). The following conditions are equivalent for every Kothe matrix $A=\left(a_{k}\right)_{k \geq 1}$.

(1) $\lambda_{1}(I, A)$ is nuclear.

(2) For every $k \geq 1$, there exist $m \geq k$ and $\beta \in \ell_{1}(I)$ such that $a_{k} \leq \beta a_{m}$.

(3) The inclusion $\lambda_{1}(I, A) \subset c_{0}(I, A)$ is a lattice isomorphism.

PROOF. (1) if and only if (2). The proof of this equivalence is similar to [14, Theorem 3.6.4], where the countable case is considered.

(2) implies (3). It is only necessary to prove that $c_{0}(I, A)$ is included in $\lambda_{1}(I, A)$. Take $\alpha=\left(\alpha_{i}\right)_{i \in I} \in c_{0}(I, A)$ and fix $k \geq 1$. From the hypothesis there exist $m \geq k$ and $\beta \in \ell_{1}(I)$ such that $a_{k} \leq \beta a_{m}$. Then

$$
\sum_{i \in I}\left|\alpha_{i}\right| a_{i, k} \leq \sum_{i \in I}\left|\alpha_{i}\right| \beta_{i} a_{i, m} \leq \sup \left\{\left|\alpha_{i}\right| a_{i, m}: i \in I\right\} \sum_{i \in I} \beta_{i}
$$

and hence $\alpha \in \lambda_{1}(I, A)$.

(3) implies (2). For every $k \geq 1$ there exist $m \geq k$ and a constant $C>0$ such that $\|\alpha\|_{k, 1} \leq C\|\alpha\|_{m, \infty}$, for all $\alpha \in c_{0}(I, A)$. Now, if $\|\alpha\|_{m, \infty} \leq 1$, then $\alpha / C \in U_{k, 1}$ (the unit ball of the seminorm $\|\cdot\|_{k, 1}$ ). Thus we have $|\langle\alpha, \gamma\rangle| \leq C$, for all $\gamma \in U_{k, 1}^{\circ}$. Hence, $\sup _{\|\alpha\|_{m, \infty} \leq 1}|\langle\alpha, \gamma\rangle| \leq C$, for all $\gamma \in U_{k, 1}^{\circ}$. In particular, for $a_{k} \in U_{k, 1}^{\circ}$ we have

$$
\sup _{\|\alpha\|_{m, \infty} \leq 1}\left|\left\langle\alpha, a_{k}\right\rangle\right| \leq C \text {. }
$$

Finally, define $\beta=\left(a_{i, k} / a_{i, m}\right)_{i \in l}$, where we agree that $0 / 0=0$. By using standard arguments it is easy to see that $\sup _{\|\alpha\|_{m . \infty} \leq 1}\left|\left\langle\alpha, a_{k}\right\rangle\right|=\sum_{i \in I} \beta_{i}$. Thus $\beta \in \ell_{1}(I)$ by (5) and clearly $a_{k} \leq \beta a_{m}$. This finishes the proof. 
REMARK 2.3. According to condition (2) in the above lemma, we see that there can be no nuclear spaces $\lambda_{1}(I, A)$ with uncountable $I$. Indeed, every family $a_{k}$ has only countably many non-zero entries (since this is true for $\beta$ ), and hence $I$ can be chosen countable. In particular, $\lambda_{1}(I, A)$ must be separable. Combining this observation with Lemma 2.5 we obtain that every discrete AL-space must be separable. That is, it cannot contain an uncountable disjoint system of discrete elements, as we point out with the following lemma.

LEMMA 2.8. Let E be a discrete Fréchet lattice with the Lebesgue property. The following conditions are equivalent:

(1) E is separable.

(2) Every subset $A \subset E$ of pairwise disjoint discrete elements is countable.

Proof. (1) implies (2). By the hypothesis we can take $0<e \in E$ a weak order unit. Let $A=\left\{e_{i}\right\}_{i \in I}$ be a subset of $E$ of pairwise disjoint discrete elements. Now, consider $x_{i}:=\inf \left\{\left|e_{i}\right|, e\right\}>0$ for all $i \in I$, and the set $B=\left\{x_{i}\right\}_{i \in I}$ of pairwise disjoint discrete elements. Observe that $0<x_{i} \leq e$ for all $i \in l$. It is not difficult to see that $\left(\sum_{i \in J} x_{i}\right)_{J \in \mathscr{P}_{f}(l)}$ is a positive increasing net order-bounded by $e$. By the Lebesgue property it follows that $\sum_{i \in I} x_{i}$ is convergent, so that $I$ must be countable.

(2) implies (1). According to Remark 2.1 and the hypothesis, take a maximal disjoint system $\left\{e_{n}\right\}_{n \in \mathbb{N}}$ consisting of positive discrete elements. Then Lemma 2.3 assures that $E$ is the closed linear span of the set $\left\{e_{n}\right\}_{n \in \mathbb{N}}$. Hence, $E$ is separable.

THEOREM 2.9. Let $E$ be a Fréchet lattice. The following conditions are equivalent:

(1) E is nuclear.

(2) $E$ is both an $A L$ and an AM-space.

ProOF. (1) implies (2). By results of Kōmura-Koshi (Lemma 2.1 and Lemma 2.6) we know that $E$ must be a discrete AL-space. By applying Lemma $2.5 E$ is lattice isomorphic to $\lambda_{1}(I, A)$ for a certain Köthe matrix $A$. Hence, $\lambda_{1}(I, A)$ is nuclear and, by Lemma 2.7 , it is lattice isomorphic to $c_{0}(I, A)$. Then, the space $E$ is lattice isomorphic to $c_{0}(I, A)$ and so it is an AM-space.

(2) implies (1). As the AL-spaces enjoy the Lebesgue property, the space $E$ must be discrete. Let $\left\{e_{i}\right\}_{i \in I}$ be a maximal disjoint system of discrete elements. Consider an increasing sequence $\left(\|\cdot\|_{k}\right)_{k}$ of lattice seminorms defining the topology of $E$ and satisfying condition (1). Also consider another increasing sequence $\left(\|\cdot\|_{k}^{\prime}\right)_{k}$ of lattice seminorms defining the topology of $E$ and satisfying condition (2) and such that both systems verify

$$
\|x\|_{k} \leq\|x\|_{k}^{\prime} \leq\|x\|_{k+1}, \quad \text { for all } x \in E \text { and } k \geq 1 .
$$


Define the Köthe matrices $A=\left(a_{k}\right)_{k \geq 1}$, where $a_{i, k}=\left\|e_{i}\right\|_{k}$ for all $i \in I$ and $B=$ $\left(b_{k}\right)_{k \geq 1}$, where $b_{i, k}=\left\|e_{i}\right\|_{k}^{\prime}$ for all $i \in l$. By using (6), it is clear that the Köthe spaces $c_{0}(I, A)$ and $c_{0}(I, B)$ are identical as Fréchet lattices. On the other hand, by applying Lemma 2.5 and Lemma $2.4, E$ is lattice isomorphic to $\lambda_{1}(I, A)$ and also to $c_{0}(I, B)$. Thus the inclusion from $c_{0}(I, A)$ onto $\lambda_{1}(I, A)$ is a lattice isomorphism. By Lemma 2.7 we have that $\lambda_{1}(I, A)$ is nuclear, and hence the space $E$ is also nuclear.

\section{References}

[1] C. D. Aliprantis and O. Burkinshaw, Locally solid Riesz spaces, Pure and Appl. Math. 76 (Academic Press, Orlando, Florida, 1978).

[2] - Positive operators, Pure and Appl. Math. 119 (Academic Press, Orlando, Florida, 1985).

[3] T. Ando, 'Banachverbände und positive Projektionen', Math. Z. 109 (1969), 121-130.

[4] K. G. Grosse-Erdmann, 'Lebesgue's theorem of differentiation in Fréchet lattices', Proc. Amer. Math. Soc. 112 (1991), 371-379.

[5] A. Grothendieck, Produits tensoriels topologiques et espaces nucléaires, Mem. Amer. Math. Soc. 16 (Amer. Math. Soc., Providence, RI, 1955).

[6] S. Kakutani, 'Concrete representation of abstract (L)-spaces and the mean ergodic theorem', Ann. of Math. (2) 42 (1941), 523-537.

[7] —, 'Concrete representation of abstract (M)-spaces', Ann. of Math. (2) 42 (1941), 994-1024.

[8] Y. Kōmura and S. Koshi, 'Nuclear vector lattices', Math. Ann. 163 (1966), 105-110.

[9] W. A. Luxemburg and A. C. Zaanen, Riesz spaces I (North-Holland, Amsterdam, 1971).

[10] R. Meise and D. Vogt, Introduction to functional analysis, Oxford Graduate Texts in Math. 2 (Clarendon Press, Oxford, 1997).

[11] A. Pietsch, Nuclear locally convex spaces, Ergebnisse der Math. und ihrer Grenzgebiete 66 (Springer, Berlin, 1972).

[12] M. N. Popa, 'Un critère de nucléarité pour des treillis', C. R. Acad. Sc. Paris Ser. A-B 269 (1969), 355-356.

[13] Y. C. Wong, 'Characterizations of the topology of uniform convergence on order-intervals', Hokkaido Math. J. 5 (1976), 164-200.

[14] _-, Schwartz spaces, nuclear spaces and tensor products, Lecture Notes in Math. 726 (Springer, Berlin, 1979).

[15] Y. C. Wong and F. K. Ng, 'Nuclear and AL-spaces', Southeast Asian Bull. Math. J. 5 (1981), 45-58.

Dpto. Matemática Aplicada II

Escuela Superior de Ingenieros

Camino de los Descubrimientos, $\mathbf{s} / \mathbf{n}$ 41092-Sevilla

Spain

e-mail: anfercar@esi.us.es
Dpto. Matemática Aplicada II Escuela Universitaria Politécnica c/ Virgen de África, 7 41011-Sevilla

Spain

e-mail: naranjo@platero.eup.us.es 
